\title{
PROYECTO FIGARO: PLATAFORMA PARA LA GESTIÓN INTEGRAL Y EFICIENTE DEL RIEGO
}

\author{
Martínez Gimeno, M.A. (1) (P), Martínez Alzamora, F. (2), Jiménez-Bello, M.A. (3), \\ Royuela. A. (4), Manzano, J. (5), Intrigliolo, D.S.(6)
}

\begin{abstract}
${ }^{1}$ Becaria Formación Profesorado Universitario. Centro de Edafología y Biología Aplicada del Segura. Consejo Superior de Investigaciones Científicas, Campus Universitario de Espinardo 30100, Espinardo, Murcia magimeno@cebas.csic.es

${ }^{2}$ Catedrático de la Universitat Politècnica de València, Instituto Universitario de Ingeniería del Agua y del Medio Ambiente, Departamento de Ingeniería Hidráulica y Medio Ambiente, Camino de Vera, s/n 46022 Valencia. fmartine@hma.upv.es
\end{abstract}

${ }^{3}$ Investigador Contratado de la Universitat Politècnica de València, Instituto Universitario de Ingeniería del Agua y del Medio Ambiente, Departamento de Ingeniería Hidráulica y Medio Ambiente, Camino de Vera, s/n 46022 Valencia. mijibar@dihma.upv.es

${ }^{4}$ Profesor Titular de la Universitat Politècnica de València, Centro Valenciano de Estudios sobre el Riego, Departamento Ingeniería Rural y Agroalimentaria, Camino de Vera, s/n 46022 Valencia. aroyuela@agf.upv.es

${ }^{5}$ Profesor Contratado Doctor de la Universitat Politècnica de València, Centro Valenciano de Estudios sobre el Riego, Departamento Ingeniería Rural y Agroalimentaria, Camino de Vera, s/n 46022 Valencia. juamanju@agf.upv.es

${ }^{6}$ Científico Titular. Centro de Edafología y Biología Aplicada del Segura. Consejo Superior de Investigaciones Científicas, Campus Universitario de Espinardo 30100, Espinardo, Murcia dintri@cebas.csic.es

\section{Resumen}

El consumo de agua y energía en el ámbito del riego en parcela es una cuestión de amplio interés y consecuencias para el medio ambiente y la economía. La solución a este doble reto exige la implantación del denominado "riego de precisión". En el presente trabajo se presenta la plataforma informática en desarrollo FIGARO (Flexible and Preclse IrriGation PIAtform to Improve FaRm Scale Water PrOductivity), la cual forma parte de un proyecto financiado por la Unión Europea dentro del $7^{\circ}$ Programa Marco, en el ámbito "Precision technologies to improve irrigation management and increase water productivity in major water-demanding crops in Europe" (KBBE.2012.1.1-03). El objetivo principal es desarrollar una herramienta de soporte a la toma de decisiones, que permita a los agricultores realizar programaciones de riego dirigidas a reducir el uso de agua, energía y fertilizantes, a la vez que se maximiza la productividad de las cosechas. La puesta en marcha y validación de la plataforma FIGARO se está llevado a cabo en diferentes países, con cultivos y condiciones climáticas diversas. Los primeros resultados de la campaña de 2014 en cultivos de cítrico y vid, sugieren incrementos de la productividad del agua con ahorros próximos al $30 \%$ de agua y del $36 \%$ de energía a nivel de comunidad de regantes. 


\begin{abstract}
Water and energy consumptions at plot level irrigation are issues of broad interest with an important environmental and economic impact. The solution to this double challenge requires the implementation of "precise irrigation". In this work is shown the under developing software system FIGARO (Flexible and Preclse IrriGation PIAtform to Improve FaRm Scale Water PrOductivity), which is part of a project co-funded by the European Commission under the $7^{\text {th }}$ Framework Programme, in the scope of "Precision technologies to improve irrigation management and increase water productivity in major water-demanding crops in Europe" (KBBE.2012.1.1-03). The aim is to develop a tool to support decision-making for end users that let them perform irrigation scheduling with the aim of reducing water, energy and fertilizers consumptions, as well as yield crop is maximized. Implementation and validation of the FIGARO platform is being performed in several countries with different crops and weather conditions. First results in the year 2014, in citrus and grapevine crops, suggest increases in water productivity with savings close to $30 \%$ of water and $36 \%$ of energy at Water User Association level.
\end{abstract}

\title{
1- Introducción. Objetivo del trabajo
}

El incremento de la demanda de agua, causada por el crecimiento de la población mundial, junto con el calentamiento global que agrava las condiciones de sequía, ha producido la disminución de los recursos hídricos. Maximizar la eficiencia del uso del agua en el ámbito del riego en parcela, responsable del $70 \%-80 \%$ del consumo total de agua en climas semiáridos como el de España, es la base para la sostenibilidad ambiental. Adicionalmente, en la mayor parte de los sistemas de riego no se dispone de cota suficiente para realizar la distribución del agua por gravedad, por lo que es necesario el empleo de grupos de bombeo que aporten al sistema la presión necesaria para su correcto funcionamiento. En estos casos, el riego no sólo supone consumo de agua, sino que implica un importante consumo energético con graves consecuencias económicas y medioambientales. La solución a este doble reto exige la implantación del denominado "riego de precisión", como herramienta de gran potencial para minimizar el consumo de recursos. En el presente trabajo se presenta la plataforma informática FIGARO (Flexible and Preclse IrriGation PIAtform to Improve FaRm Scale Water PrOductivity), en cuyo desarrollo participan 17 socios de 8 países (Israel, Reino Unido, Dinamarca, Holanda, Portugal, Italia, Grecia y España). El objetivo principal es crear una herramienta de soporte a la toma de decisiones, que permita a los agricultores realizar programaciones de riego dirigidas a reducir el uso de agua, energía y fertilizantes, a la vez que se maximiza la productividad de las cosechas. La plataforma, actualmente, se encuentra en su fase final de desarrollo e implementación en campo.

\section{2- Bases de la plataforma para la gestión del riego}

La plataforma FIGARO está compuesta por un conjunto de subsistemas que recogen datos ambientales y de los cultivos, entre otros. Con ellos se alimentan una serie de módulos que configuran el sistema de soporte a la toma de decisiones (DSS), el cual proporciona recomendaciones fiables a los usuarios sobre cuánto y cuándo deben regar para optimizar los recursos. El sistema es modular y flexible, por lo que podría actualizarse a lo largo del tiempo con la implementación de nuevas tecnologías y nuevos modelos agronómicos. Los usuarios (agricultores, comunidades de regantes, técnicos...) podrán 
personalizar el sistema para satisfacer sus necesidades individuales de una forma fácil y accesible.

En la Figura 1 queda representada la arquitectura del sistema. En el siguiente apartado se detallan cada uno de los datos, modelos y módulos que emplean para su funcionamiento.

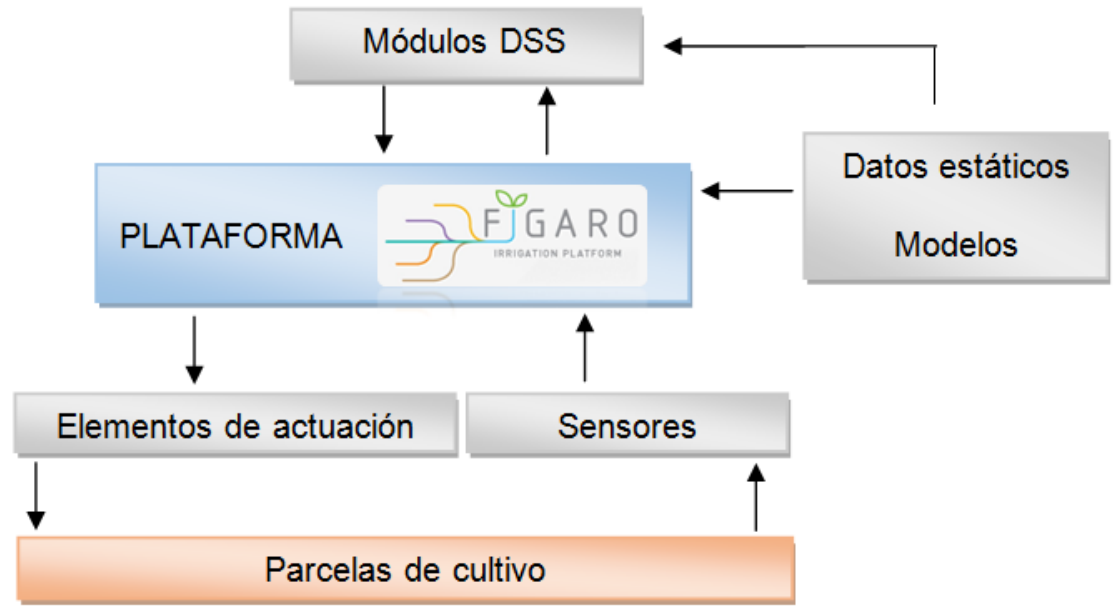

Figura 1. Arquitectura de la plataforma FIGARO

\section{1- Estructura de la plataforma}

La plataforma FIGARO basa su funcionamiento en el uso simultáneo de bases de datos y diversos modelos de optimización, integrados en servidores propios desarrollados en el proyecto. Concretamente, los datos estáticos, los datos dinámicos procedentes de los sensores y los elementos de actuación, son almacenados y mostrados al usuario a través de una interfaz gráfica denominada uManage. Ésta a su vez, alimenta a la plataforma AquaSafe, que integra los diferentes módulos dirigidos a optimizar la producción de los cultivos y el consumo de agua y energía. En la Figura 2 se puede ver, a modo de ejemplo, una de las pantallas del sistema uManage.

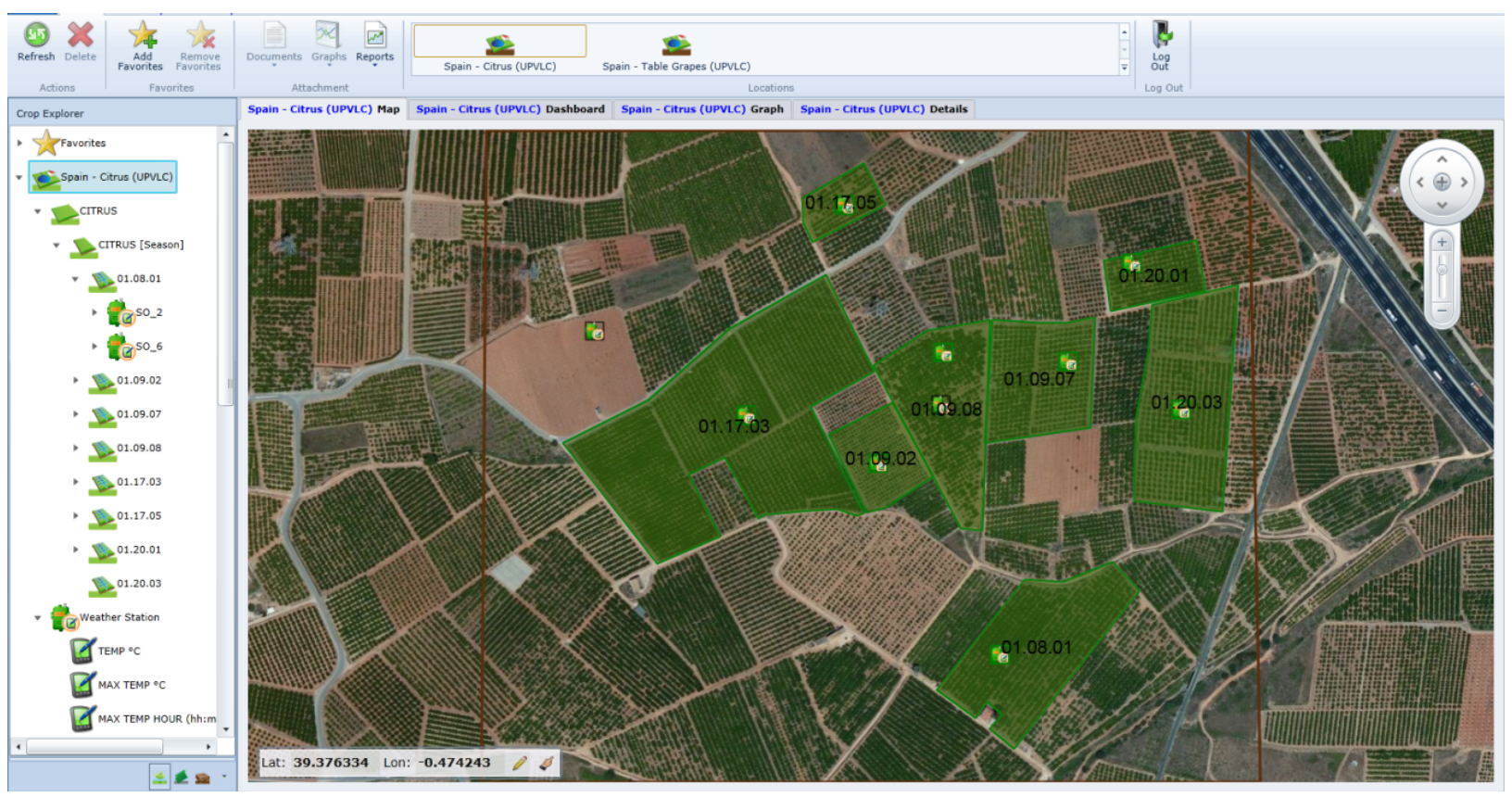


Figura 2. Vista del sistema uManage. Detalle visor y desglose de parcelas y sensores.

- Modelo de simulación del crecimiento de los cultivos

Son diversos los modelos empleados en la plataforma para la simulación y seguimiento de las producciones en base a las recomendaciones de riego y fertilización. Cada uno de los integrantes del consorcio que forma FIGARO emplea el modelo que más se ajusta a las características de los cultivos y climatología. Los principales modelos utilizados por ahora son: Daisy (Heidmann et al., 2008) para cultivo de patata, Mohid-Land (Galvão et al, 2004 y Chambel-Leitão et al, 2011) para maíz, y Aquacrop (Land and Water Division of FAO) para cultivos herbáceos en general. A diferencia de los socios del proyecto que trabajan con cultivos herbáceos (tomate, patata, maíz y algodón), la parte que se desarrolla en España, se centra cultivos leñosos. Concretamente se trabaja con cítricos y vid, no quedando éstos recogidos en ninguno de los modelos anteriores. Por ello, la metodología empleada para la optimización de recursos para los cultivos leñosos se ha adaptado en base a sus características intrínsecas. Los dos grandes ejes que permiten calcular los recursos a emplear y realizar seguimientos de la evolución de los cultivos son los siguientes:

Modelos para la optimización de la programación del riego.

Las necesidades de riego para los cultivos leñosos, tanto de los cítricos como de la vid, se basan en las recomendaciones que el Servicio de Tecnología del Riego del Instituto Valenciano de Investigaciones Agrarias (IVIA) proporciona semanalmente mediante su servicio web. Estas recomendaciones proporcionan valores que se ajustan a las características de los cultivos y a sus condiciones climáticas. Concretamente el cálculo relativo a las necesidades de riego en cítricos se basa en un estudio llevado a cabo por Ginestar y Castel (1996), el cual define la reducción relativa del rendimiento en relación con la evapotranspiración de cultivo en Clementina de Nules para clima mediterráneo. De la misma forma, el modelo de cálculo de las necesidades en vid se basa en estudios que determinan el coeficiente de cultivo para uva (Williams y Ayars, 2004; y López-Urrea et al 2006). Adicionalmente para optimizar el riego, al tiempo que se minimizan las posibles mermas en la producción a nivel de cada parcela, estas recomendaciones se ajustan considerando los valores semanales del potencial de tallo medidos con cámara de presión, el contenido de agua del suelo medido mediante sondas humedad multisensor, y la previsión meteorológica.

Integración de la información suministrada mediante teledetección.

Los modelos de estimación de necesidades de riego anteriores necesitan ciertos parámetros, como la cubierta vegetal, para su implementación y seguimiento. Mediante el procesado de ortofotos e imágenes de satélite de alta resolución (RapidEye) se puede obtener este parámetro para cada una de las parcelas objeto de estudio. Del mismo modo, se generan mapas de evapotranspiración real aplicando métodos de balance de energía en superficie (SEBAL, Bastiaanssen et al. 1998a, Bastiaanssen et al. 1998b). Para este fin se utilizan las imágenes proporcionadas por Landsat 8 con una frecuencia temporal de 16 días. Esta información también es utilizada para estimar las cantidades teóricas de riego y compararlas con los registros de los contadores con el fin de evaluar la gestión mediante la producción de mapas de indicadores de gestión.

- Modelos de simulación del comportamiento de la red hidráulica

Las características específicas de las zonas de cultivo mediterráneas, normalmente asociadas en Comunidades de Regantes para el suministro del agua de riego a través de redes colectivas, requieren un especial tratamiento de las programaciones para optimizar el 
uso conjunto de los recursos. Para ello se utilizan modelos matemáticos de las redes, que simulan el comportamiento hidráulico de éstas, con el fin de asegurar el suministro bajo las condiciones de garantía requeridas. El modelo hidráulico está preparado para mantenerse calibrado mediante los datos proporcionados por los sistemas SCADA, los cuales se alimentan de sensores como caudalímetros, contadores, transductores de presión y analizadores de energía. Con la implementación de estrategias de optimización de los turnos de riego (Jiménez-Bello et al. 2010, Jiménez-Bello et al 2015) se consigue aportar a los cultivos el agua previamente estimada, con el mínimo consumo energético.

\section{2 - Puesta en marcha y validación de la plataforma.}

La puesta en marcha y validación de la plataforma FIGARO se está llevado a cabo simultáneamente en diferentes países, con cultivos y condiciones climáticas diversas. Como se ha comentado anteriormente, los casos de estudio del equipo español, y que se presentan en esta comunicación, se centran en dos cultivos leñosos. Por un lado, se dispone de parcelas de cítricos ubicadas en el sector XI de la Comunidad de Regantes de Picassent y por otro, una parcela experimental de vid localizada en Cheste, ambas en la provincia de Valencia. La organización y diversidad de las parcelas de cítricos gestionadas conjuntamente con la Comunidad de Regantes, ha permitido realizar diferentes programaciones para comparar la eficiencia de la plataforma FIGARO en condiciones reales. El caso concreto de la parcela de vid, está aportando información de los diferentes tratamientos (cuatro variantes que incluyen riego deficitario) a nivel de parcela, bajo condiciones de tratamiento muy controladas. Ambas zonas de estudio se complementan para mostrar la disparidad en el uso de los recursos en función del nivel de organización del sistema agrícola. Durante los años 2013 y 2014 se ha llevado a cabo la instalación y puesta en marcha de los equipos, así como el desarrollo de las programaciones de riego en base a las directrices planteadas en el proyecto.
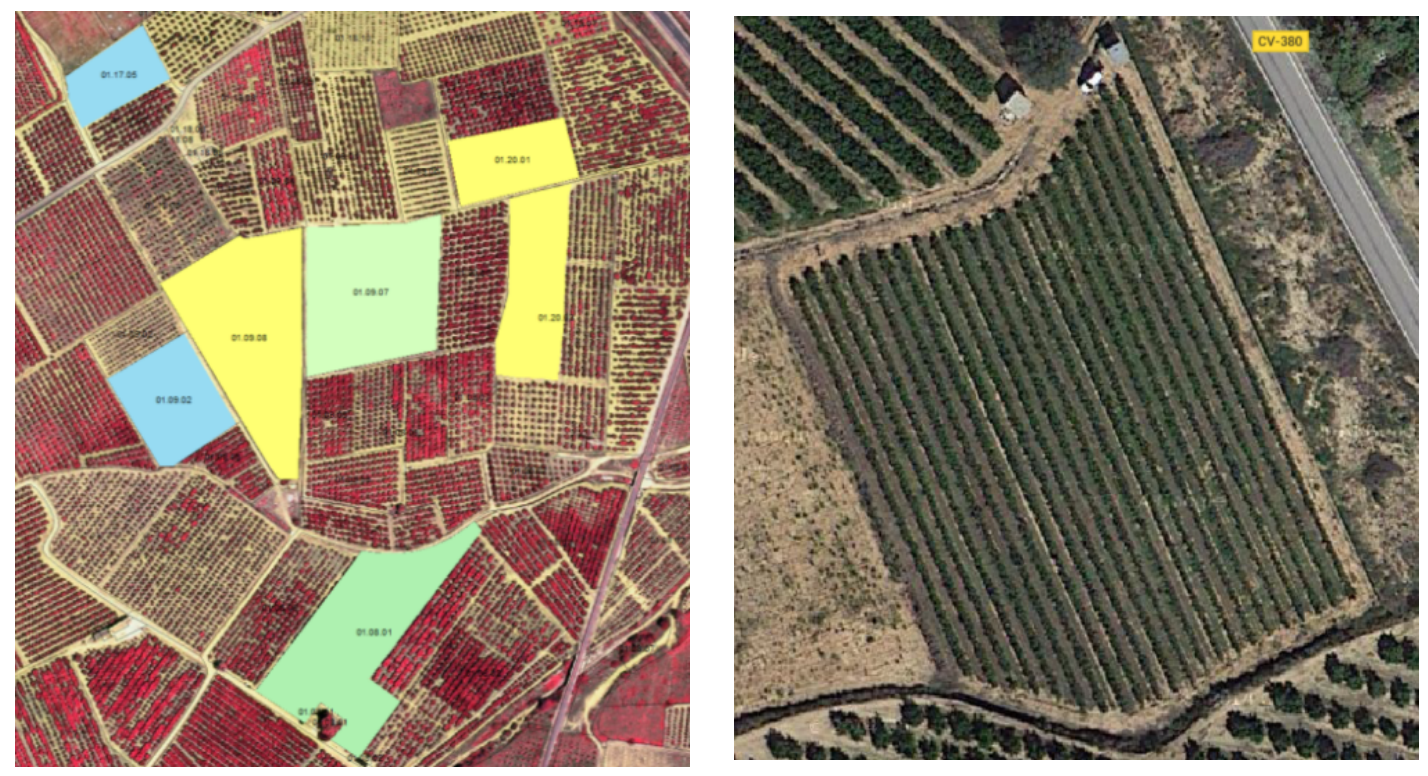

Figura 3. Izquierda, parcelas de cítricos, Picassent (Valencia) 39²2'46.36"N, 0²8'19.92"O. Derecha, parcela de vid, Cheste (Valencia) $39^{\circ} 33^{\prime} 28.7^{\prime \prime} \mathrm{N}, 0^{\circ} 42^{\prime} 22.6^{\prime \prime} \mathrm{O}$.

Así mismo, se ha monitorizado el funcionamiento de la red hidráulica de la Comunidad de Regantes de Picassent mediante la adquisición en tiempo real de los datos 
relativos a consumos eléctricos, velocidades de giro de los grupos de bombeo, niveles de balsas, caudales suministrados, presiones en puntos estratégicos, consumos de las tomas en parcela y la programación de riego llevada a cabo. Ello está permitiendo mantener el modelo hidráulico calibrado de manera continua, con el fin de realizar la programación de los turnos de riego en base a estrategias de optimización que garanticen un consumo energético mínimo, a la vez que se aporta el agua necesaria determinada por los modelos agronómicos a través de la plataforma.

A pesar de haber sufrido un año con una demanda evaporativa muy alta, los primeros resultados de la campaña de 2014, sugieren el incremento de la productividad del agua en las dos zonas de estudio empleando las directrices desarrolladas por la plataforma FIGARO. En el caso de los cítricos, se han alcanzado ahorros del $30 \%$ en agua, sin mermas en la producción, y ahorros del $36 \%$ de energía a nivel de comunidad de regantes. En la parcela piloto de vid, se ha conseguido notables ahorros de agua, de alrededor del $40 \%$, mediante la aplicación de técnicas de riego deficitario.

\section{3- Resultados y conclusiones}

Con el fin de dar respuesta a los retos medioambientales y económicos que hoy día son incuestionables en el ámbito de la agricultura, la plataforma FIGARO se convertirá en una herramienta completa para el riego de precisión integrado. A través del uso de sensores que controlan el continuo suelo - planta - atmósfera, de las herramientas de previsión meteorológica, de los sistemas de información geográfica, junto con la teledetección y otros sistemas de control, el sistema presentará a los usuarios un protocolo óptimo de riego aplicable tanto a nivel de parcela como a nivel de comunidad de regantes.

\section{4- Agradecimientos}

Este trabajo ha sido financiado por Unión Europea dentro del $7^{\circ}$ Programa Marco, en el ámbito "Precision technologies to improve irrigation management and increase water productivity in major water-demanding crops in Europe" (KBBE.2012.1.1-03).

\section{5- Bibliografía}

Bastiaanssen, W. G. M., Menenti, M., Feddes, R. A. \& Holstlag, A. A. M. (1998a). A remote sensing surface energy balance algorithm for land (SEBAL). Part 1. Formulation. J. Hydrol. 212-213, 198-212.

Bastiaanssen, W. G. M., Pelgrum, H., Wang, J., Ma, Y., Moreno, J., Roerink, G. J. \& van der Wal,T. (1998b) The surface energy balance algorithm for land (SEBAL): Part 2. Validation. J. Hydrol. 212-213, 213-229.

Chambel-Leitão, P., Almeida, C., Jauch, E., Trancoso, R., Neves, R., \& Leitão, J. C. (2011). Evapotranspiration forecast using SWAT model and weather forecast model. SWAT International conference. Toledo. España.

Galvão, P. B., Neves, R., Silva, A., Leitão, P. C., \& Braunchweig, F. (2004). Integrated watershed modeling. European Space Agency ESA SP, 549, 187-190. 
Ginestar, C. \& Castel, J.R. (1996). Response of young 'Clementine' citrus trees to water stress during different phenological periods. J. Hort. Sci., 71, 551-559.

Heidmann, T., Tofteng, C., Abrahamsen, P., Plauborg, F., Hansen, S., Battilani, A., \& Vacek, J. (2008). Calibration procedure for a potato crop growth model using information from across Europe. Ecological modelling, 211(1), 209-223.

Jiménez-Bello M.A, Martínez Alzamora, F., Bou Soler, V. \&. Bartoli Ayala, H.J. (2010). Methodology for grouping intakes of pressurised irrigation networks into sectors to minimise energy consumption, Biosystems Engineering 105 (2010), pp. 429-438.

Jiménez-Bello M.A.,Royuela A., Manzano J., García Prats A. \& Martinez Alzamora, F. (2015). Methodology to improve water and energy use by proper irrigation scheduling in pressurised networks. Agricultural Water Management 149, 91-101.

López-Urrea, R., Montoro, A., Mañas, F., López-Fuster, P., \& Fereres, E. (2012). Evapotranspiration and crop coefficients from lysimeter measurements of mature 'Tempranillo' wine grapes. Agricultural Water Management, 112, 13-20.

Williams, L. E. \& Ayars, J. E. (2005). Water use of Thompson Seedless grapevines as affected by the application of gibberellic acid (GA 3) and trunk girdling-practices to increase berry size. Agricultural and Forest Meteorology, 129(1), 85-94. 